\title{
PARECERISTAS 2014
}

Ada Augusta Celestino Bezerra

Universidade Tiradentes

Adair Mendes Nacarato

Universidade São Francisco

Ademir Donizete Caldeira

Universidade Federal de São Carlos

Adir Casaro Nascimento

Universidade Católica Dom Bosco

Adriana Lia Friszman Laplane

Universidade Estadual de Campinas

Agueda Bernardete Bittencourt

Universidade Estadual de Campinas

Alexandre Fernandez Vaz

Universidade Federal de Santa Catarina

Alice Maria Figueira Reis da Costa Fundação de Apoio à Escola Técnica

do Estado do Rio de Janeiro

Alvanize Valente Fernandes Ferenc

Universidade Federal de Viçosa

Ana Luiza Bustamante Smolka

Universidade Estadual de Campinas

Anderson Jonas das Neves

Universidade Estadual Paulista Júlio de Mesquita Filho

André Picanço Favacho

Universidade Federal de Minas Gerais

Angela Maria Martins

Fundação Carlos Chagas

Universidade Cidade de São Paulo

Antonio Carlos Rodrigues de Amorim

Universidade Estadual de Campinas

Antônio Sérgio Alfredo Guimarães

Universidade de São Paulo

Cancionila Janzkovski Cardoso

Universidade Federal de Mato Grosso

\section{Carla Alexandra da Silva Moita Minervino}

Universidade Federal da Paraíba

Carla Maria Rezende Rodrigues

Associação Brasileira de Psicopedagogia

\section{Carlos Eduardo Vieira}

Universidade Federal do Paraná
Carlos Frederico Bernardo Loureiro

Universidade Federal do Rio de Janeiro

Carlos Roberto Jamil Cury

Pontifícia Universidade Católica de Minas Gerais

Carmen Campoy Scriptori

Universidade Estadual de Campinas

Celi Espasandin Lopes

Universidade Cruzeiro do Sul

Celso Sánchez Pereira

Universidade Federal do Estado do Rio de Janeiro

Christian Muleka Mwerwa

Universidade Federal de Mato Grosso do Sul

Cláudia Ribeiro Bellochio

Universidade Federal de Santa Maria

Claudia Maria Costa Alves de Oliveira

Universidade Federal Fluminense

Claudia Maria de Lima

Universidade Estadual Paulista Júlio de Mesquita Filho

Cláudia Maria Ribeiro

Universidade Federal de Lavras

\section{Claudio Roberto Baptista}

Universidade Federal do Rio Grande do Sul

Cynthia Paes de Carvalho

Pontifícia Universidade Católica do Rio de Janeiro

Daliana Löffler

Universidade Federal de Santa Maria

David Mesquita Costa

Centro Universitário Adventista de São Paulo

Deisi Sangoi Freitas

Universidade Federal de Santa Maria

Denise Meyrelles de Jesus

Universidade Federal do Espírito Santo

Diana da Veiga Mandelert

Universidade do Estado do Rio de Janeiro

Diana Gonçalves Vidal

Universidade de São Paulo

Doris Pires Vargas Bolzan

Universidade Federal de Santa Maria

Dulce Maria Filgueira de Almeida

Universidade de Brasília 


\section{Ecleide Cunico Furlanetto}

Universidade Cidade de São Paulo

Edith Ione dos Santos Frigotto

Universidade Federal Fluminense

Elenilton Vieira Godoy

Centro Universitário Fundação Santo André

Eliane Ribeiro Andrade

Universidade Federal do Estado do Rio de Janeiro

Eliane Rose Maio

Universidade Estadual de Maringá

Elionaldo Fernandes Julião

Universidade Federal Fluminense

Elisabeth Brandão Schmidt

Universidade Federal do Rio Grande

Elizeu Clementino de Souza

Universidade do Estado da Bahia

Emília da Trindade Prestes

Universidade Federal da Paraíba

Emília Freitas de Lima

Universidade Federal de São Carlos

Emilio Celso de Oliveira

Universidade Paulista

Eulina Maria Leite Nogueira

Universidade Federal do Amazonas

Eurize Caldas Pessanha

Universidade Federal de Mato Grosso do Sul

Fábio Delano Vidal Carneiro

Faculdade Sete de Setembro

Flaminio de Oliveira Rangel

Universidade Federal de São Paulo

Flávia Brocchetto Ramos

Universidade de Caxias do Sul

Flávia da Silva Ferreira Asbahr

Universidade Estadual Paulista Júlio de Mesquita Filho

Flavia Maria Chiara Candusso

Universidade Federal da Bahia

Fúlvia Maria de Barros Mott Rosemberg

Fundação Carlos Chagas

Gelson Vanderlei Weschenfelder

Complexo de Ensino Superior de Cachoeirinha

Geraldo Romanelli

Universidade de São Paulo

Gideon Borges dos Santos

Fundação Oswaldo Cruz

Gilda Cardoso de Araújo

Universidade Federal do Espírito Santo
Gilka Elvira Ponzi Girardello

Universidade Federal de Santa Catarina

Giovanna Marafon

Pontifícia Universidade Católica do Rio de Janeiro

Graziela Giusti Pachane

Universidade Federal do Triângulo Mineiro

Heloísa Helena Pimenta Rocha

Universidade Estadual de Campinas

Inês Barbosa de Oliveira

Universidade do Estado do Rio de Janeiro

Isabel Moreira Macedo

Universidade do Minho

Ivana Maria Schnitman

Centro Universitário Estácio da Bahia

Ivanilde Apoluceno de Oliveira

Universidade do Estado do Pará

Jacques Therrien

Universidade Estadual do Ceará

Universidade Federal do Ceará

Jaime Giolo

Universidade Federal da Fronteira Sul

Jarbas Santos Vieira

Universidade Federal de Pelotas

Jefferson Mainardes

Universidade Estadual de Ponta Grossa

Joana Paulin Romanowski

Pontifícia Universidade Católica do Paraná

João dos Reis Silva Junior

Universidade Federal de São Carlos

João Feres Júnior

Universidade do Estado do Rio de Janeiro

João Ferreira de Oliveira

Universidade Federal de Goiás

Johanna Milena Rey Herrera

Centro de Investigación y de Estudios Avanzados del Instituto Politécnico Nacional

Jorge Manuel Gorostiaga

Universidad Nacional de General San Martín

José Licínio Backes

Universidade Católica Dom Bosco

José Sérgio Fonseca de Carvalho

Universidade de São Paulo

Julio Groppa Aquino

Universidade de São Paulo

Katia Maria Penido Bueno

Fundação Educacional Lucas Machado 
Kátia Oliver de Sá

Faculdade Regional da Bahia

Laura Cristina Vieira Pizzi

Universidade Federal de Alagoas

Leandro Belinaso Guimarães

Universidade Federal de Santa Catarina

\section{Leda Scheibe}

Universidade Federal de Santa Catarina

Universidade do Oeste de Santa Catarina

Liliana Soares Ferreira

Universidade Federal de Santa Maria

Luani de Liz Souza

Universidade do Estado de Santa Catarina

Lucídio Bianchetti

Universidade Federal de Santa Catarina

Luciene Fontão

Secretaria Municipal de Educação de Florianópolis

\section{Ludmila Thomé de Andrade}

Universidade Federal do Rio de Janeiro

Luis Marcelo Carvalho

Universidade Federal de Lavras

Luiza Pereira Monteiro

Universidade Estadual de Goiás

Magna do Carmo Silva Cruz

Universidade Federal de Pernambuco

Malvina do Amaral Dorneles

Universidade Federal do Rio Grande do Sul

Márcia Ondina Vieira Ferreira

Universidade Federal de Pelotas

Marcio Rodrigo Vale Caetano

Universidade Federal do Rio Grande

Márcio Santos de Santana

Universidade Estadual de Londrina

Marcos Villela Pereira

Pontifícia Universidade Católica do Rio Grande do Sul

Marcus Aurélio Taborda de Oliveira

Universidade Federal de Minas Gerais

Maria Abádia da Silva

Universidade de Brasília

Maria Amélia da Costa Lopes

Universidade do Porto

Maria Antônia de Souza

Universidade Tuiuti do Paraná

Maria Apparecida Campos Mamede Neves

Pontifícia Universidade Católica do Rio de Janeiro

Maria Auxiliadora Soares Padilha

Universidade Federal de Pernambuco
Maria Cândida Müller

Universidade Federal de Rondônia

Maria Clara Di Pierro

Universidade de São Paulo

Maria Cristina de Carvalho Cascelli de Azevedo Universidade de Brasília

Maria Cristina Soares de Gouvêa

Universidade Federal de Minas Gerais

Maria da Conceição Passeggi

Universidade Federal do Rio Grande do Norte

Maria das Graças Vieira

Universidade Federal de Pernambuco

Maria José Pires Barros Cardozo

Universidade Federal do Maranhão

Maria Luiza Canedo

Pontifícia Universidade Católica do Rio de Janeiro

Maria Rita de Almeida Toledo

Universidade Federal de São Paulo

Maria Suzana de Stefano Menin

Universidade Estadual Paulista Júlio de Mesquita Filho

Maria Sylvia Cardoso Carneiro

Universidade Federal de Santa Catarina

Maria Teresa Santos Cunba

Universidade do Estado de Santa Catarina

Marilda Gonçalves Dias Facci

Universidade Estadual de Maringá

Marilia Gouvea de Miranda

Universidade Federal de Goiás

Marilyn Aparecida Errobidarte de Matos

Instituto Federal de Educação, Ciência e

Tecnologia de Mato Grosso do Sul

Marilza Vanessa Rosa Suanno

Universidade Federal de Goiás

Mário Luiz Neves de Azevedo

Universidade Estadual de Maringá

Marlene Ribeiro

Universidade Federal do Rio Grande do Sul

Marli Elisa Dalmaso Afonso de André

Pontifícia Universidade Católica de São Paulo

Marta Maria de Araújo

Universidade Federal do Rio Grande do Norte

Martha Marandino

Universidade de São Paulo

Meily Assbú Linhales

Universidade Federal de Minas Gerais

Milton Rosa

Universidade Federal de Ouro Preto 
Mirtes Ribeiro de Lira

Universidade de Pernambuco

Monica Ribeiro Silva

Universidade Federal do Paraná

Moysés Kublmann Júnior

Universidade São Francisco

Nelson Cardoso Amaral

Universidade Federal de Goiás

Núbia Pereira Paiva

Universidade do Sudoeste da Bahia

Odalina Emiko Aoki Alves

Defensoria Pública de Abaetetuba

Olgaíses Cabral Maués

Universidade Federal do Pará

Pablo Silva Machado Bispo dos Santos

Universidade Federal Fluminense

Paola Basso Menna Barreto Gomes Zordan

Universidade Federal do Rio Grande do Sul

Paula Regina Costa Ribeiro

Universidade Federal do Rio Grande

Paulo Marcelo Marini Teixeira

Universidade Estadual do Sudoeste da Bahia

Regina Tereza Cestari de Oliveira

Universidade Católica Dom Bosco

Renata Maraschin

Universidade de Passo Fundo

Renato José de Oliveira

Universidade Federal do Rio de Janeiro

Rita Rezende Vieira Peixoto Migliora

Pontifícia Universidade Católica do Rio de Janeiro

Roberta Gurgel Azzi

Universidade Estadual de Campinas

Rodrigo Pelloso Gelamo

Universidade Estadual Paulista Júlio de Mesquita Filho

Romualdo Luiz Portela de Oliveira

Universidade de São Paulo

Rosa Fátima de Souza

Universidade Estadual Paulista Júlio de Mesquita Filho

Rosa Maria Hessel Silveira

Universidade Federal do Rio Grande do Sul

Rosalba Maria Cardoso Garcia

Universidade Federal de Santa Catarina
Rosália Maria Duarte

Pontifícia Universidade Católica do Rio de Janeiro

Rosângela Tenório de Carvalho

Universidade Federal de Pernambuco

Rosemeire Reis da Silva

Universidade Federal de Alagoas

Sandra Maria Zákia Lian Sousa

Universidade de São Paulo

Saraí Patrícia Schmidt

Universidade Feevale

Selva Guimarães Fonseca

Universidade Federal de Uberlândia

Sergio Paulino Abranches

Universidade Federal de Pernambuco

Silvia Cristina Yannoulas

Universidade de Brasília

Silvio Donizetti de Oliveira Gallo

Universidade Estadual de Campinas

Simone de Lucena Ferreira

Universidade Federal de Sergipe

Sirlei de Lourdes Lauxen

Universidade de Cruz Alta

Sonia Barbosa Camargo Igliori

Pontifícia Universidade Católica de São Paulo

Talita Vidal Pereira

Universidade do Estado do Rio de Janeiro

Tatiana de Pino Albuquerque Maranhão

Ministério da Ciência, Tecnologia e Inovação

Telma Ferraz Leal

Universidade Federal de Pernambuco

Vânia Gomes Zuin

Universidade Federal de São Carlos

Vera Lúcia Gaspar da Silva

Universidade do Estado de Santa Catarina

Vera Maria Ferrão Candau

Pontifícia Universidade Católica do Rio de Janeiro

Vera Maria Nigro de Souza Placco

Pontifícia Universidade Católica de São Paulo

Zaia Brandão

Pontifícia Universidade Católica do Rio de Janeiro 